\title{
RAFDS: Remote Abnormality and Fall Detection System for Assisting Older Persons
}

\author{
Yosra Ismail ${ }^{1}$, Mariem Kallel ${ }^{2}$, Loay Ismail ${ }^{3}$ \\ Department of Computer Science and Engineering, College of Engineering, Qatar University, Doha, Qatar ${ }^{1,2,3}$
}

\begin{abstract}
One of the main problems facing the public health is the injury that happens due to older persons falling, as these injuries can be fatal for them. Fast and proper medical interventions are crucial to reduce the serious consequences on their health. In this paper, a system is designed to continuously monitor vital signs and motion of older persons, and automatically detects incidents of abnormal vital signs as well as the fall of unattended older persons, and, accordingly, sends an alarm message to the concerned caregivers, to provide the needed help in the shortest delay possible. The proposed system is composed of three major modules; firstly the sensing module (SM), which continuously measures the acceleration of the monitored person, as well as vital signs of the monitored persons, such as the temperature and the heart pulse rate. Secondly, the decision making module (DMM) which is a processing element that receives the sensing signals from the sensors used in SM, analyzes them, then makes a decision if an abnormality or a fall is detected or not. Finally, if the DMM detects a fall, it sends this decision signal to the alarm module (AM), which sends SMS messages to the concerned caregivers, whose cell phone numbers, are pre-configured in the AM.
\end{abstract}

Keywords: Fall detection, heart pulse rate, body temperature, abnormality detection, remote detection.

\section{INTRODUCTION}

Referring to a United Nation's press release on the world the older people so that they can instantaneously give the population in 2013 [1], the current world population of needed help in case of occurrence of falls. This 7.2 billion is projected to increase by almost one billion requirement is crucial to reduce the effect of the falls. But people within the next 12 years, reaching 8.1 billion in on the other hand a caregiver can only handle one or a 2025 and 9.6 billion in 2050. Life expectancy has very few number of older people, so a huge number of increased for the world as a whole from 47 years in 1950- caregivers are needed as the number of older people 1955 up to 69 years in 2005-2010. Furthermore, life increases. Such huge number of caregivers cannot be expectancy is expected to continue rising to reach 70 years achieved in reality. Thus, an assisting fall detection in 2045-2050 and 78 years in 2095-2100. Therefore, a system, that can automatically detect if an older person phenomenon of population ageing is occurring throughout falls and gives an alarm to the caregiver, is needed to the whole world. For example, in 2013, the proportion of complement the job of the caregivers.

older persons in the developed countries had exceeded that The significance of introducing an automatic fall detection of children (23\% versus 16\%). In 2050, it is expected that system has been implicitly stated in the Qatar National the older persons' percentage is to be double that of Vision 2030 Report, under the Human Development pillar: children (32\% versus 16\%) in developed countries. While "To improve the health of Qatar's population, Qatar in least developed countries it is expected to have $19 \%$ aspires to develop an integrated system for health care, older persons versus $22 \%$ of children.

The UN World Health Organization gave high attention to the welfare of the older persons and they pointed that one of the prominent risks that may face older people is the accidental falls. "The frequency of fall increases with age, where approximately $30-50 \%$ of people living in longterm care institutions fall each year, and $40 \%$ of them experienced recurrent fall. The rate of hospital admission due to falls for people at the age of 60 and older in countries like Canada and United Kingdom range from 1.6 to 3.0 per 10,000 population." [2]. It was found that falls and their consequent injuries are a major public health problem that needs proper medical attention. Moreover, it was found that fatal falls rates increase exponentially with age for both sexes, with highest rates for ages of 85 and over [2].

A problem that may arise due to the population ageing and its related problem of accidental falls is the increasing need of availability of caregivers and nurses to fully attend managed according to world-class standards, that will meet the needs of existing and future generations to provide an increasingly healthy and lengthy life for all of its citizens." [3].

Existing fall detection systems are still having several challenges such as limited range of operation being restricted to indoor environment, need of expensive infrastructure for reliable detection, and obtrusiveness of the detection system as the individual has to wear special setting of sensors [4].

\section{RELATED WORK}

Accidental falls are seriously harmful events for the older persons and more than $33 \%$ of people aged 65 years and above experience one fall every year [4]. As a consequence of falls, an individual may be harmed both physiologically and psychologically. This can be in the form of an injury of various types, a limited activity, and an uneasiness of living independently and fear of falling 
[4]. Thus, reducing the risk of a person's harm from fall comes at the top of the safety goals for developed and developing countries. Here comes the importance of designing and implementing an automatic Fall Detection System that detects the fall of unattended older persons and sends an alarm message to the concerned caregivers, to provide the needed help in the shortest delay possible.

Fall detection systems can be classified into three main approaches [4]: (1) Computer vision and image processing based approach, in which the movement of the person is monitored in real time through a video capturing system and some algorithms are applied to recognize the posture of the person [5][6][7][8]; (2) Acoustic based approach, in which a fall is detected by analysing the frequency components caused by the vibrations associated with the fall [9][10][11]; (3) Worn sensor based approach, in which kinematic sensors such as accelerometers are worn by the individual to distinguish between a fall and a normal activity of daily life [4][12][13][14][15][16].

In each of the above mentioned approaches, different types of sensing modules, decision making modules, and alarm modules are used. These modules may differ with respect to (1) their suitability of usage indoor and/or outdoor; (2) their complexity of deployment and maintenance; (3) their need for a special infra structures; (4) and finally their cost.

As for the types of sensing modules, single or multiple cameras can be used for computer vision based approach, microphones and/or vibration sensors can be used for acoustic based approach, and accelerometers and/or gyroscopes can be used for worn sensor based approach.

As for decision making modules, a dedicated PC is needed for both computer vision based approach and acoustic based approach, where the PC is needed for the analysis of images to recognize the patient's posture and for the analysis of the acoustic sensors signals to detect a fall. On the other hand, worn sensor based can make use of either a remote PC or an on-board microcontroller to analyse the signals produced by the accelerometers and/or gyroscopes. As for the alarm modules, all approaches can use a telecommunication device such as a $3 \mathrm{G}$ device to send SMS messages and video streams to the concerned caregivers, when a fall is detected.

In the literature, most of the FDS systems are based on the worn sensor based approach, as it has an edge with respect to the other approaches that can be summarized as follows:

- Can be easily used in normal living environments.

- Can be used in both indoor and outdoor.

- Has a relatively low cost.

- Does not need special infrastructures for deployment. However, its main challenge is the battery life to be used in this type of standalone system and its obtrusive nature as it requires the older person to wear special setting of sensors.

On the other hand, the computer vision based approach has the advantage of being unobtrusive and can be used in determining the position of the falling victim in a precise manner. So, the challenge is to combine the different approaches to make use of their exclusive advantages to obtain an accurate and reliable FDS system.
In this paper, a wearable remote fall detection system (FDS) is presented, characterized by its light weight and small size. This system is augmented with a module for monitoring vital signs, such as body temperature and heart pulse rate. Moreover, this system is intelligent enough to detect both fall incidents and abnormal conditions, based on vital signs, and provide alarms in case of detecting falls and/or abnormalities, constituting a Remote Abnormality and Fall Detection System (RAFDS).

\section{III.OVERVIEW OF THE PROPOSED SYSTEM}

In this paper, we will present a real time RAFDS system to reduce the harmful consequence of falling of older persons through an early detection of the fall accident and an instantaneous alarming system, to notify the caregivers about the incident in real time. The proposed RAFDS system will be primarily designed to be used indoors and we will present a study of how to extend it to be used to monitor vital signs, such as heart pulse rate and body temperature.

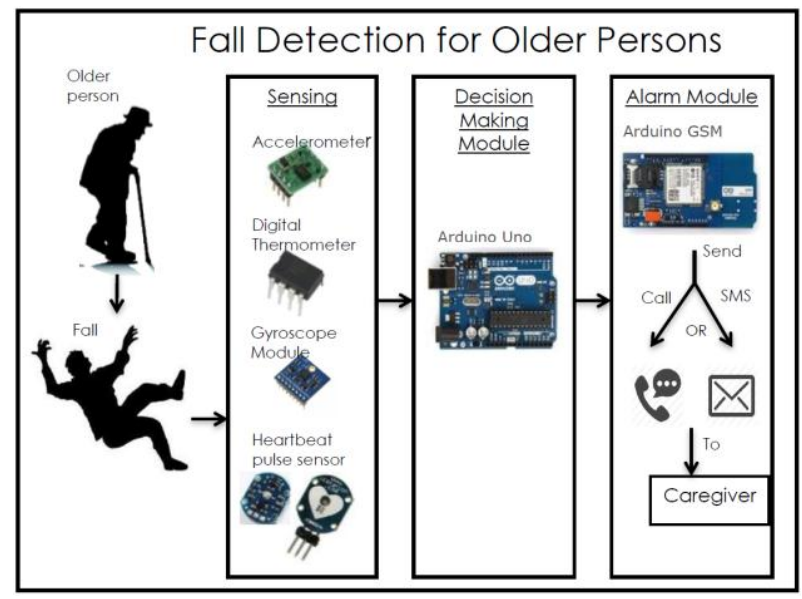

Fig.1 Components of the RAFDS

The RAFDS system will be composed of the following modules, as shown in figure 1:

a) Sensing module (SM) which continuously measures the acceleration, heart pulse rate and temperature of the monitored person. To build this module, the following types of sensors are needed: a tri-axis accelerometer to continuously measure the acceleration of the monitored subject in three dimensions, a temperature sensor to continuously measure body temperature and heart pulse rate sensor to continuously capture the heart pulse rate of the person. A GPS module can be also used, if needed, to capture the person's outdoors position.

b) Decision making module (DMM) which receives the sensing signals from the different sensors, analyze them, then make a decision if a fall or abnormal vital sign is detected or not. It is composed of a processing element which can be in the form of a microcontroller like Arduino or PIC, or a digital signal processor (DSP) like TMS320F28335-based DSPs, which can be used in both indoors and outdoors. The DMM can be connected to the SM either locally through direct connections, or remotely through a wireless communication, such as ZigBee-based communication. 
c) Alarm module (AM) which receives a decision signal from the DMM that a fall or an abnormal vital sign is detected, and the AM, in its turn, sends an SMS message to the concerned caregivers, whose cell phone numbers are pre-configured in the AM. The AM is composed of either a GSM module or even a $3 \mathrm{G}$ module if a video stream needs to be established.

In this paper, we will present the design and implementation of the RAFDS SM, DMM and AM modules, and finally show experimental results of the proposed system.

\section{IV.DESIGN OF THE RAFDS}

The RAFDS system consists of three Modules: sensing module, decision making module and alarm module. As for the sensing module, it consists of three sensors: accelerometer sensor (MMA7455) [17], Digital Thermometer sensor (Ds1620) [18] and heart-rate pulse sensor (HRPS) [19] to detect any sudden change happened of heart rate. These sensors continuously measure the data from the sensors on periodic bases. As for the decision-making module, the Arduino UNO microcontroller used to capture and process the data measured by the sensors. As for the alarm module, there are two kinds of alarms. There is an alarm for the elderly person and alarm for the caregiver. As for the alarm for the elderly person, a speaker and a button are used to cancel any false detection, either from the accelerometer when false fall detection is detected or from the heart rate pulse sensor if the user is doing a physical activity. As for the alarm for the caregiver, the GSM shield sendsan SMS message to the caregiver, whenever there is a sudden change happened to the elderly person to help him. Based on the functional requirements and the practical design constraints, we decided about the hardware components and the software design that will control the whole system.

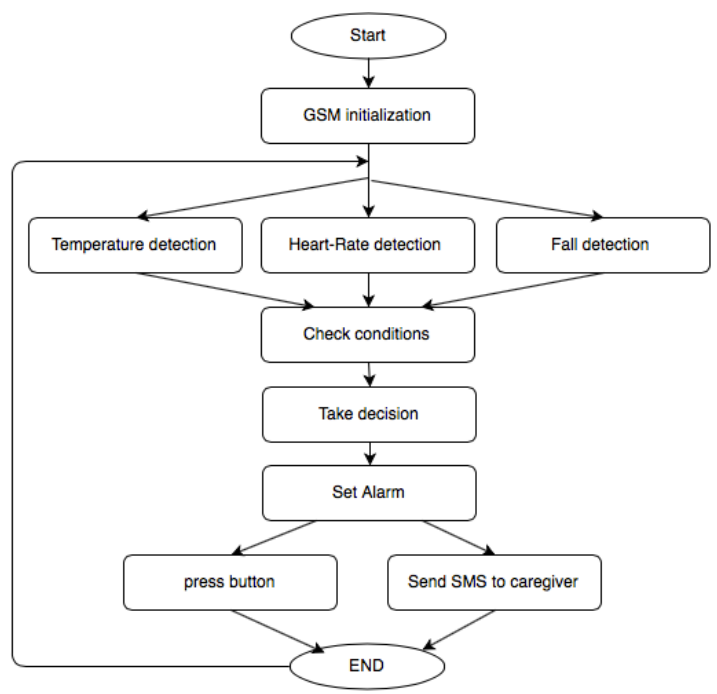

Fig. 2 High Level Architecture Flow chart Temperature Abnormality Detection:

The microcontroller will read the data from the temperature sensor and then compare it to the threshold value.
The normal body temperature should be $37^{\circ} \mathrm{C}$.

However, normal body temperature depends on from which part of the body the temperature measurement is taken. So, to make things easier we assumed that the normal body temperature is in the range of $37^{\circ} \mathrm{C} \pm 1{ }^{\circ} \mathrm{C}$, i.e. between $36^{\circ} \mathrm{C}$ and $38^{\circ} \mathrm{C}$. The data read by the sensor will be compared to this threshold; if it goes out of this range then the system will send an SMS to the caregiver with the temperature measured.

\section{A. Heart Rate Anomaly Detection:}

The heart rate is the number of times the heart beats per minute (BPM). A normal heart rate depends on the individual's age, body size, fitness level, heart conditions, emotions and specially the type of the current physical condition. It is proven that some accurate calculations are used to compute the heart rate of the body depending on the age

Maximum heart rate $=206.9-(0.67 \times$ Age $)[20]$

According to the Centers for Disease Control and Prevention, for moderate-intensity physical activity, the target heart rate of the resting person is 50 percent to 70 percent of his maximum heart rate. If the person is doing some physical activities, its target rate is 70 percent to 85 percent of his maximum heart rate.

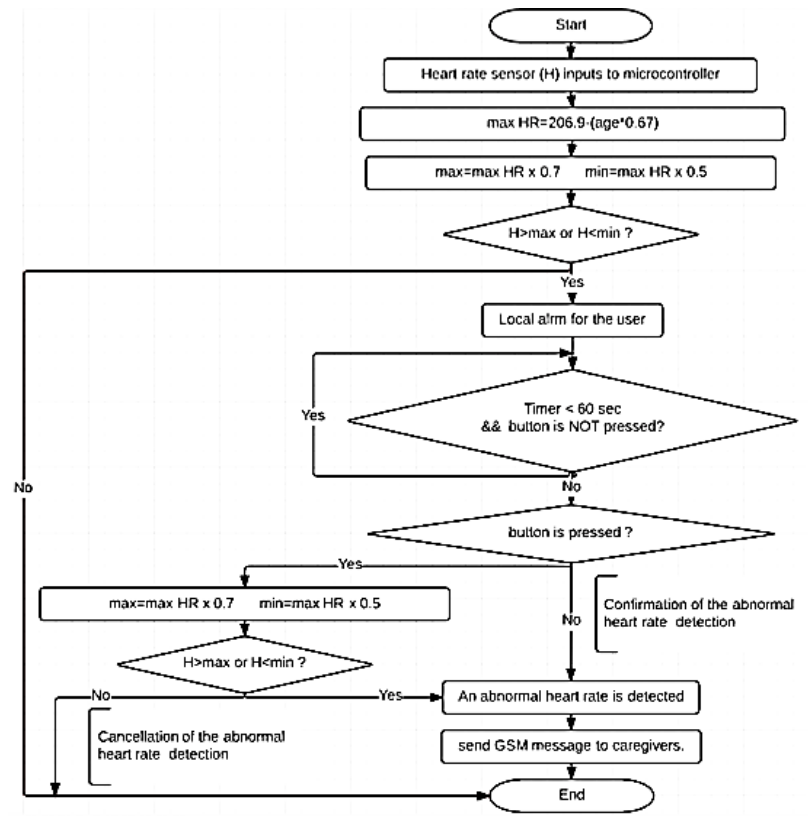

Fig.3Heart Rate Anomaly Detection

As an example of a 50-year-old person, its maximum heart rate is $206.9-(0.67 \times 50)=173.4 \mathrm{BPM}$. The target heart rate in rest is from $170 \times 0.50=85 \mathrm{BPM}$ to $170 \times 0.70=$ 119 BPM, and from $170 \times 0.70=119$ BPM to $170 \times 0.85=$ 144 BPM when doing a physical activity.

As shown in figure 3 , the system is designed to first define the normal region of the heart rate of the resting person by calculating the maximum and minimum values. Then, the actual heart rate of the person will be compared to the predefined region. If it is out of the limits of the safe region, an abnormal heart rate will be detected for the resting person. Then an alarm will be launched to the user 
itself to inform him about the decision. If he doesn't press on the cancel button within a predefined time, the abnormal heart rate detection will be confirmed, and the system will send a message to caregivers informing them about the problem. However, if the user is doing a physical activity, he should press on the button within a predefined time to inform the system about that. In this case, the system will define the normal region of the heart rate of the Non-resting person by calculating the maximum and minimum value. Then, the actual heart rate of the person will be again compared to the predefined region. If it is out of the limits of the safe region, an abnormal heart rate will be detected for the Non-resting person and a message will be sent to care givers. The system uses a for loop to define a limited time where the user can press on the button; right after the abnormal heart rate detection of the resting person, the button will be activated for only 60 seconds in order to let the time for the user to press it. After the 60 seconds, the button will no more function. As a result, the detection will be confirmed and the system will move to the alarm module.

\section{B. Fall Detection:}

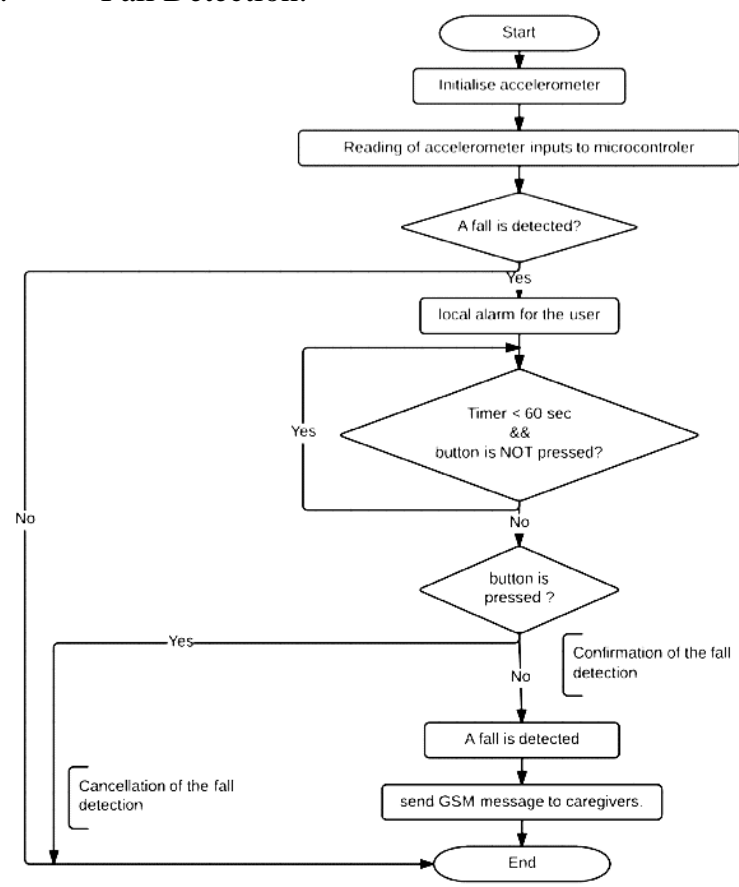

Fig.4 Fall Detection

The fall detection system, shown in figure 4, starts by the accelerometer that keeps sensing the acceleration of the user and inputting the value to the microcontroller. Then, the microcontroller decides if a fall occurs based on some thresholds. The fall is detected by detecting the state of weightlessness that occurs while the body is in a free fall. This state can be detected using accelerometers, as will be discussed in the implementation section.

In case of falling, the button will be used to guarantee the accuracy and the confirmation of the fall before sending a message to caregivers and announce the fall detection. If the decision-making module detects a fall, an alarm will be launched to the user itself to inform him about the decision. If the user has truly fallen and the decision of the microcontroller is correct, he probably will be fainting and unconscious and ignoring the alarm. If the detection of the microcontroller is false, the alarm announces the false detection to the user, who is able to cancel it by pressing the button within a predefined time, and prevent the system from moving to the alarm module and send a message to caregivers informing them about the fall.

When the user alarm starts, the button will be activated for only 60 seconds in order to let the time for the user to press it. After the 60 seconds, the button will no more function. As a result, the fall detection will be confirmed and the system will move to the alarm module and send a message to caregivers to inform them about the fall.

\section{IMPLEMENTATION OF THE RAFDS}

The system continuously reads the output of the sensors on periodic bases. The microcontroller compares these data with the conditions of each sensor to decide if there is any abnormal change or not. If any abnormal change is detected an alarm launched so that the elderly person can stop it by pressing the button if it was a false alarm otherwise a SMS message sent to the caregiver to provide help to the elderly person as shown on figure 5 .

\section{A. Arduino UNO microcontroller}

The microcontroller used in the system is the Arduino UNO board which is a microcontroller board based on theATmega328. It can be simply powered with external DC battery. It has 14 digital input/output pins as well as 6 analog input pins that can be used to interface with different types of external components. Also, it has a low price compared to its speed.

\section{B. Arduino GSM Shield}

The Arduino GSM shield was chosen as the GSM modem, to send/receive SMS messages between the older system and the caregivers. It just needs a SIM card to be connected to the local mobile network. The shield uses only three pins of the Arduino board, in addition to the 5 volts $(5 \mathrm{~V})$ and ground (GND) pins. GSM RX (pin 2) is connected to pin 2 in Arduino board, GSM TX (pin 3) is connected to pin 3 in Arduino board and Modem Reset (pin 7) to pin 7 in board.

\section{MMA7445 three-axis accelerometer}

The MMA7445 three-axis accelerometer wasused for measuring the acceleration of the body. It can be easily interfaced with Arduino UNO board. Its registers can be programmed to work in different modes, with different selectable levels of sensitivity ( $2 \mathrm{~g}, 4 \mathrm{~g}$ or $8 \mathrm{~g}$ ). Moreover, it needs low voltage between $2.4 \mathrm{~V}$ to $3.6 \mathrm{~V}$. The accelerometer was used to detect weightlessness by programming it to work in the "level detection" mode, in which an interrupt is generated when the acceleration in all directions ( $\mathrm{x}, \mathrm{y}$ and $\mathrm{z}$ ) falls below a certain threshold (say $0.25 \mathrm{~g}$ ). Once the interrupt is generated, it is handled by the microcontroller to notify a detection of a fall. MMA7455 accelerometer has eight DIP pins. Only six pins are used in this sensor. It requires voltage power (5V) and Ground connection (GND). The CLK pin in MMA7455 is 
connected to A5 in Arduino board and DATA in heart rate pulse readings faster than any other pulse sensor. MMA7455 is connected to A4 in board. In the code, the Pulse sensor requires low power voltage either $3 \mathrm{~V}$ or $5 \mathrm{~V}$.

INT1 and INT2 pins were initialized to be connected to A0 and A1 respectively. The MMA7455 is interfaced to the Arduino through the Serial Peripheral Interface (SPI) bus.

\section{Ds1620 Temperature sensor}

Ds1620 is a digital temperature sensor that measures temperatures from $-55^{\circ} \mathrm{C}$ to $+125^{\circ} \mathrm{C}$ using a band gapbased temperature sensor. The data can be read or written using three wire serial interface; DQ, CLK, RST. The user can reset it because it has thermostatic settings. It requires a power voltage $2.7 \mathrm{~V}$ as minimum voltage and $5.5 \mathrm{~V}$ as maximum voltage. It doesn't require any external components such as capacitors or resistors, so that it can easily connected to Arduino UNO microcontroller. The temperature sensor Ds 1620 has 8 DIP pins. It requires power voltage $(5 \mathrm{~V})$ and Ground connection (GND). The first three pins are the output pins that will be used to read the temperature. These pins (DQ, CLK, and RST) should be connected to digital pins. DQ is connected to pin 6 , CLK is connected to pin 5 and RST is connected to pin 4.

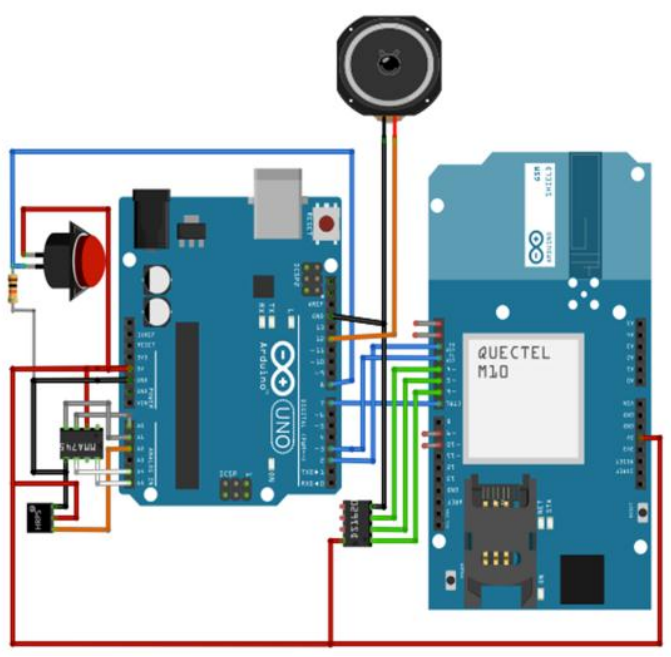

Fig.5 Electric Circuit of Full System

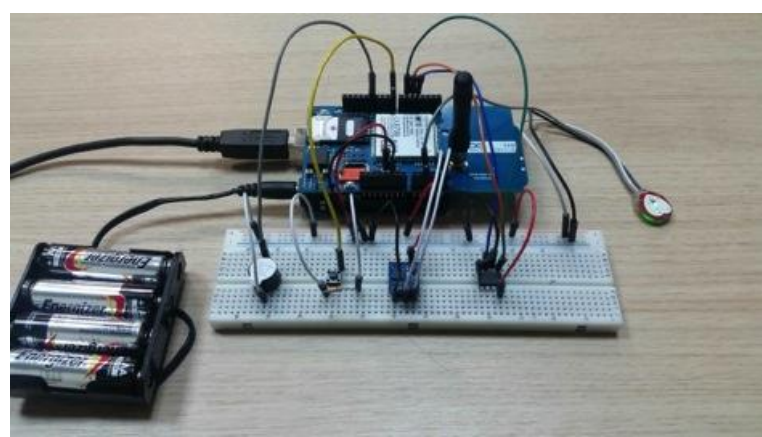

Fig.6 Wiring of Full Syste

\section{E. Heart Rate Pulse Sensor}

Heart rate pulse sensor for Arduino was used. It is directly connected to the Arduino UNO board without using any external connections or components. It provides a reliable

\section{F. Speaker}

The speaker used accepts the TTL levels and can be driven using PWM with different frequencies and duty cycles. The speaker is fed by an analog Pulse Width Modulation (PWM) signal, generated by the Arduino microcontroller.

\section{G. Button}

SPST (Single Pole Single Throw) is the simplest type of on-off switches. It has two terminals; they can be connected together or disconnected from each other. The push button is connected to the ground by a $10 \mathrm{k} \mathrm{Ohm}$ resistor and to the VCC. The upper pin of the button that faces the resistor is connected to pin 8 of the Arduino.

\section{H. Integrated System}

All the components will be combined together to act as a full system, as shown in figure 5 and 6 . Three sensors are used; accelerometer to detect fall, temperature sensor to measure the temperature and heart rate pulse sensor to measure the heart beats and time between beats. A GSM shield to connect the device with a local network for sending messages to the caregiver. A Button that will be used by user to delete the false alarm and a speaker to inform the user that asudden change happened.

\section{VI.EXPERIMENTAL RESULTS}

The following table shows the sensitivity of the system in detecting fall and anomalies in both heart rate and temperature. The fall detection sensitivity was $90 \%$ at $0.25 \mathrm{~g}$ threshold. It was observed that the sensitivity may increase by the decrease of the threshold below $0.25 \mathrm{~g}$, but the number of false positive increases considerably. The sensitivity of abnormal heart rate detection was $86.67 \%$ due to the in accuracy of the heart pulse rate sensor. The sensitivity of the abnormal temperature detection was $80 \%$ due to the inappropriateness of the Ds1620 to measure the body temperature. A special setting was designed and implemented to keep the sensor in continuous contact with the person body, but the ambient temperature was a considerable source of disturbance.

TABLE I EXPERIMENTAL RESULTS

\begin{tabular}{|c|c|c|c|}
\hline $\begin{array}{c}\text { Test of 30 } \\
\text { trials }\end{array}$ & $\begin{array}{c}\text { True } \\
\text { Positive } \\
\text { (TP) }\end{array}$ & $\begin{array}{c}\text { False } \\
\text { Negative } \\
\text { (FN) }\end{array}$ & $\begin{array}{c}\text { Sensitivity = } \\
\text { TP/(TP+FN) }\end{array}$ \\
\hline $\begin{array}{c}\text { Fall } \\
\text { detection }\end{array}$ & 27 & 3 & $90 \%$ \\
\hline $\begin{array}{c}\text { Abnormal } \\
\text { heart rate }\end{array}$ & 26 & 4 & $86.67 \%$ \\
\hline $\begin{array}{c}\text { Abnormal } \\
\text { temperature }\end{array}$ & 24 & 6 & $80 \%$ \\
\hline
\end{tabular}

\section{CONCLUSION}

The presented system is designed to reduce the danger that faces unattended elder people due to falls, and abnormal vital signs such as heart pulse rate and temperature. The system is capable of automatically detecting the fall and 
the abnormal signs through a set of sensors, such as accelerometer, heart pulse detector and digital thermometer, followed by a microcontroller that analyzes the data captured by the sensors, to decide the existence of a fall or abnormality in vital signs. If a fall or abnormality in vital signs is detected, an alarming SMS message will be sent through a GSM modem to the concerned caregivers. The system prototype showed acceptable results with respect to sensitivity to the occurrence of fall and abnormality in vital signs.

\section{ACKNOWLEDGMENT}

This paper was made possible by Qatar University Student Grant \# QUST-CENG-SPR-13/14-9. The statements made herein are solely the responsibility of the author[s].

\section{REFERENCES}

[1] World Population Prospects: The 2012 Revision, http://esa.un.org/unpd/wpp/Documentation/pdf/WPP2012_Press_R elease.pdf (Accessed 3rd of April 2014).

[2] WHO Global Report on Falls Prevention in Older Age, http://www.who.int/ageing/publications/Falls_prevention7March.pd $\mathrm{f}$ (Accessed 3rd of April 2014).

[3] Qatar National Vision Report, https://www.google.com/url?sa=t\&rct=j\&q=\&esrc=s\&source=web $\& \mathrm{~cd}=1 \&$ ved=0CCgQFjAA\&url=http $\% 3 \mathrm{~A} \% 2 \mathrm{~F} \% 2 \mathrm{Fwww} . q u . e d u . q a$ $\% 2$ Fpharmacy\%2Fcomponents\%2Fupcoming_events_material\%2F Qatar_National_Vision_2030.pdf\&ei=Dc8U8XKHKf9ywONroHw Bw\&usg=AFOjCNH2wLHjxxuz0L vFlolqGjrPKqbrA\&sig2=I94P DAwDGFspgQIANDEXqw\&bvm=bv.63934634,d.bGQ\&cad=rja (Accessed 3rd of April 2014).

[4] F. Hijaz, N. Afzal, T. Ahmad, and O. Hasan, "Survey of Fall Detection and Daily Activity Monitoring Techniques", IEEE proceeding, 2010 International Conference on Information and Emerging Technologies (ICIET), p. 1-6, 2010

[5] S. Miaou, P. Sung, and C. Huang, "A Customized Human Fall Detection System Using Omni-Camera Images and Personal Inform", Proceedings of 1 st Transdisciplinary Conference on Distributed Diagnosis and Mome Healthcare, (D2H2 2006), p. 3942, 2006.

[6] M. Yu, Y. Yu, A. Rhuma, S.M.R. Naqvi, L. Wang, and J.A. Chambers, "An Online One Class Support Vector Machine-Based Person-Specific Fall Detection System for Monitoring an Elderly Individual in a Room Environment", IEEE Journal of Biomedical and Health Informatics, Volume 17, Issue 6, p. 1002-1014, 2013.

[7] Y Chen, Y. Lin, and W. Fang, "A HYBRID HUMAN FALL DETECTION SCHEME", Proceedings of 2010 IEEE 17th International Conference on Image Processing, p. 3485-3488, 2010.

[8] Y. Li, K. C. Ho, and M. Popescu, "Efficient Source Separation Algorithms for Acoustic Fall Detection Using a Microsoft Kinect", IEEE TRANSACTIONS ON BIOMEDICAL ENGINEERING, VOL. 61, NO. 3, MARCH 2014

[9] A. Sixsmith, N. Johnson, "A Smart Sensor to Detect the Falls of the Elderly," IEEE Pervasive Computing, vol. 3, no. 2, p. 42-47, 2004.

[10] M. Alwan, P. Rajendran, S. Kell, D. Mack, S. Dalal, M. Wolfe, R. Felder, "A Smart and Passive Floor-Vibration Based Fall Detector for Elderly", Information and Communication Technologies, vol. 1, p. 1003-1007, 2006.

[11] M. Popescu, Y. Li, M. Skubic, M. Rantz, "An acoustic fall detector system that uses sound height information to reduce the false alarm rate", Engineering in Medicine and Biology Society, 2008. EMBS 2008. 30th Annual International Conference of the IEEE, p. 4628 $4631,2008$.

[12] C. Lan, Y. Hsueh, and R. Hu, "Real-Time Fall Detecting System Using a Tri-axial Accelerometer for Home Care", Proceedings of the International Conference on Biomedical Engineering and Biotechnology (iCBEB 2012), p. 1077-1080, 2012.

[13] S. Zhang, H. Li, P. McCullagh, C. Nugent, and H. Zheng, "A Realtime Falls Detection System for Elderly", Proceedings of the 5th Conference on Computer Science and Electronic Engineering (CEEC 2013), p. 51-56, 2013.
[14] C. Huang, C. Chiang, G. Chen, S. Hsu, W. Chu, and C. Chan, "Fall Detection System for Healthcare Quality Improvement in Residential Care Facilities", Journal of Medical and Biological Engineering, Volume 30, Issue 4, p. 247-252, 2010.

[15] F. Büsching, H. Post, M. Gietzelty, and L. Wolf, "Fall Detection on the Road", Proceedings of 2013 IEEE 15th International Conference on e-Health Networking, Applications and Services (Healthcom 2013), p.439-443, 2013

[16] S. Fang, Y. Liang, and K. Chiu," Developing a Mobile Phone-based Fall Detection System on Android Platform", Proceedings of Conference on Computing, Communications and Applications (ComComAp 2012), p. 143-146, 2012

[17] MMA7455-3-axis accelerometer datasheet, http://www.parallax.com/sites/default/files/downloads/28526MMA 7455-3-Axis-Accelerometer-Documentation-v1.1.pdf (Accessed 11 th of November 2014)

18] DS1620 digital thermometer datasheet, http://www.parallax.com/sites/default/files/downloads/604-00002 DS1620-Datasheet.pdf (Accessed 11th of November 2014).

[19] Heart rate pulse sensor datasheet,http://www.ebay.com/itm/HeartRate-Pulse-Sensor-Pulsesensor-Sensor-Module-For-Arduino-OpenSource-/371160050591 (Accessed 13th of November 2014).

[20] K A., Zimmermann, L. \& Contributor, "What is a Normal Heart Rate". livescience.com: http://www.livescience.com/42081-normalheart-rate.html, 18 December. 2013 